\title{
[FeFe] Hydrogenase based Prototype Photosensitizer- Catalyst Dyad for Hydrogen Generation under Visible Light
}

Philipp Buday, ${ }^{[a]}$ Chizuru Kasahara, ${ }^{[a, b]}$ Elisabeth Hofmeister, ${ }^{[c]}$ Daniel Kowalczyk, ${ }^{[d]}$ Micheal K. Farh, ${ }^{[a]}$ Saskia Riediger, ${ }^{\left[{ }^{[g]}\right.}$ Martin Schulz, ${ }^{[c, e]}$ Maria Wächtler, ${ }^{[c, e, f]}$ Shunsuke Furukawa, ${ }^{[b]}$ Masaichi Saito, ${ }^{* b]}$ Dirk Ziegenbalg, ${ }^{[d]}$ Stefanie Gräfe, ${ }^{[\mathrm{e}, \mathrm{f}, \mathrm{h}, \mathrm{i}]}$ Peter Bäuerle, ${ }^{[\mathrm{g}]}$ Stephan Kupfer, ${ }^{*[\mathrm{e}]}$ Benjamin Dietzek-Ivanšić, ${ }^{*[, e, f, h]}$ Wolfgang Weigand ${ }^{*[a]}$

\footnotetext{
[a] P. Buday, ${ }^{(+)}$C. Kasahara, ${ }^{(+)}$M. K. Farh, Prof. W. Weigand Institute of Inorganic and Analytical Chemistry Friedrich Schiller University Jena Humboldtstraße 8, 07743 Jena, Germany E-mail: wolfgang.weigand@uni-jena.de

[b] C. Kasahara, ${ }^{(+)}$Dr. S. Furukawa, Prof. M. Saito Department of Chemistry Graduate School of Science and Engineering, Saitama University Shimo-okubo, Sakura-ku, Saitama-city, Saitama, 338-8570, Japan E-mail: masaichi@chem.saitama-u.ac.jp

[c] E. Hofmeister, Dr. M. Schulz, Dr. M. Wächtler, Prof. B. Dietzek-Ivanšić Department Functional Interfaces Leibniz Institute of Photonic Technology Jena (Leibniz-IPHT) Albert-Einstein-Straße 9, 07745 Jena, Germany

[d] D. Kowalczyk, Prof. D. Ziegenbalg Institute of Chemical Engineering Ulm University Albert-Einstein-Allee 11, 89081 Ulm, Germany

[e] Dr. M. Schulz, Dr. M. Wächtler, Dr. S. Kupfer, Prof. S. Gräfe, Prof. B. Dietzek-Ivanšić Institute of Physical Chemistry Friedrich Schiller University Jena Helmholtzweg 4, 07743 Jena, Germany

E-mail: benjamin.dietzek@uni-jena.de; stephan.kupfer@uni-jena.de

[f] Dr. M. Wächtler, Prof. S. Gräfe, Prof. B. Dietzek-Ivanšić Abbe Center of Photonics (ACP)

Friedrich Schiller University Jena Albert-Einstein-Straße 6, 07745 Jena, Germany

[g] Prof. P. Bäuerle, S. Riediger Institute of Organic Chemistry II and Advanced Materials Ulm University Albert-Einstein-Allee 11, 89081 Ulm, Germany

[h] Prof. B. Dietzek-Ivanšić, Prof. S. Gräfe

Center for Energy and Environmental Chemistry Jena (CEEC Jena) Friedrich Schiller University Jena Philosophenweg 8, 07743 Jena, Germany

[i] Prof. S. Gräfe

Fraunhofer Institute for Applied Optics and Precision Engineering Albert-Einstein-Straße 7

07745 Jena, Germany

(+) These authors contribute equally to the work.
} 


\section{Abstract}

Inspired by the active center of the natural [FeFe] hydrogenases, we designed a compact and precious metal-free photosensitizer-catalyst dyad (PS-CAT) for photocatalytic hydrogen evolution under visible light irradiation. PS-CAT represents a prototype dyad comprising $\pi$ conjugated oligothiophenes as light absorbers. PS-CAT and its interaction with the sacrificial donor 1,3-dimethyl-2-phenylbenzimidazoline were studied by steady-state and time-resolved spectroscopy coupled with electrochemical techniques and visible light-driven photocatalytic investigations. Operando EPR spectroscopy revealed the formation of an active [ $\mathrm{Fe}^{\prime} \mathrm{Fe}^{0}$ ] species - in accordance with theoretical calculations - presumably driving photocatalysis effectively (TON $\approx 210$ ).

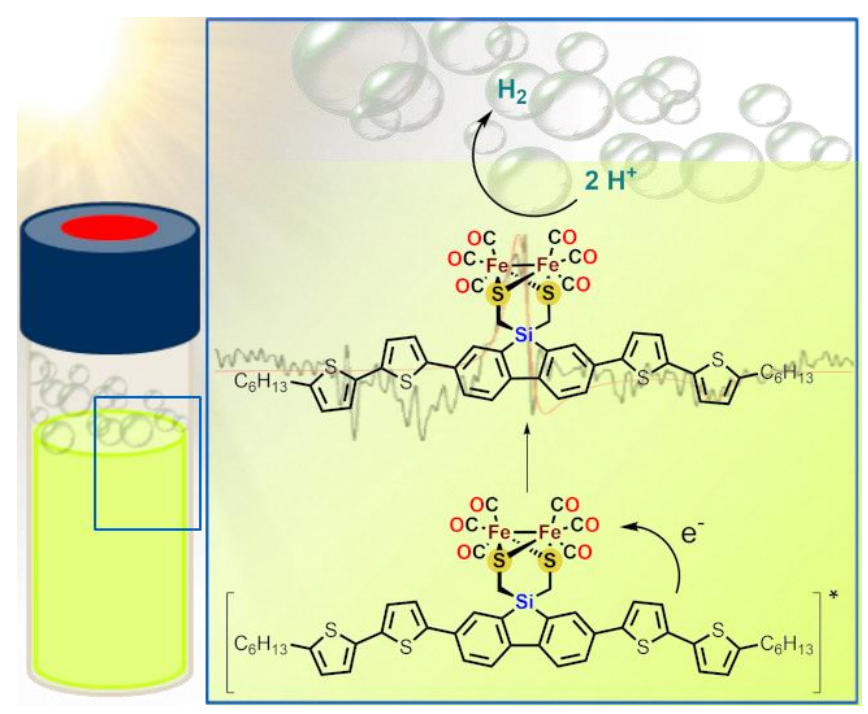


Nature provides paradigms for photocatalysis to generate solar fuels in a renewable and climate-neutral fashion. In this context, scientists have created various architectures to perform catalytic key processes, e.g. the hydrogen evolution reaction (HER), under light excitation. Commonly, $4 \mathrm{~d}$ and $5 \mathrm{~d}$ transition metals (e.g. $\mathrm{Ru}, \mathrm{Cd}, \mathrm{Re}, \mathrm{Ir}, \mathrm{Pt}$ ) play a crucial role in molecular artificial HER photocatalysis - both as light-harvesters and catalysts - due to their favourable (photo)redox chemistry and stability. ${ }^{[1]}$ However, rare abundance or undesired side effects (e.g. toxicity) hamper a larger scale application.

Nature uses earth-abundant, inexpensive reaction centers in the [FeFe] hydrogenase enzyme for HER. ${ }^{[2]}$ Inspired by the natural paradigm [FeFe] hydrogenase mimics have been reported. ${ }^{[3]}$ Such catalysts, however, often rely on photosensitizers based on precious metals. Few exceptions are given by e.g. xanthene dyes, (zinc) porphyrins ${ }^{[1,, 4]}$ or carbon $\operatorname{dots}^{[5]}$ as light harvesters. We recently reported another entirely precious metal-free example, a dibenzosilole photosensitizer directly attached to the catalyst, yielding a TON of 539 for HER under UV light $(254 \mathrm{~nm}) \cdot{ }^{[6]} \pi$-Conjugated oligothiophenes present metal-free, readily accessible and strong light absorbers in the visible spectral region. The optical and redox properties of such dyes are used e.g. in organic ${ }^{[7]}$ or dye-sensitized ${ }^{[8]}$ solar cells. Ott and colleagues combined a $\pi$ conjugated oligothiophene photosensitizer and a [FeFe] hydrogenase mimic in a cosensitized $\mathrm{NiO}$ photocathode ${ }^{[9]}$ with only minor HER yields.

We report a fully precious metal-free, visible-light absorbing, bioinspired and compact photocatalyst dyad PS-CAT as a prototype hybrid comprising $\pi$-conjugated oligothiophenes as photosensitizer for the light-driven HER. We present PS-CAT, its photocatalytic activity in the presence of the sacrificial donor 1,3-dimethyl-2-phenylbenzimidazoline $(\mathrm{BIH})$ and a detailed mechanistic characterization. The photosensitizer unit represents a major improvement over the previous reported UV-active dibenzosilole light harvester, which showed promising electron relay properties to the covalently bound catalyst, leading to fundamental changes in the photophysical and photocatalytic features.

PS-CAT combines the light-harvesting properties of $\pi$-conjugated oligothiophenes (PS) with a bioinspired [FeFe] hydrogenase mimic (CAT). It was synthesized according to Scheme 1 (also detailed in Scheme S1) from the dithioacetato compound PS. Both, PS and PS-CAT were characterized by NMR, IR spectroscopy and mass spectrometry. The molecular structure of PS-CAT (Figure 1; detailed in Figure S2 and Table S1/S2) reveals a similar binding mode compared to a previous reported, structurally related complex. ${ }^{[6]} A$ view on the ab plane shows stacking of planar PS-CAT molecules (Figure S2b). The cyclic voltammogram of PS-CAT in $\mathrm{CH}_{2} \mathrm{Cl}_{2}$ at a scan rate of $0.2 \mathrm{Vs}^{-1}$ shows a quasi-reversible reduction event at $E_{1 / 2}=-1.61 \mathrm{~V} v \mathrm{~s}$ $\mathrm{Fc}^{+} / \mathrm{Fc}$ (Figure S8a). 


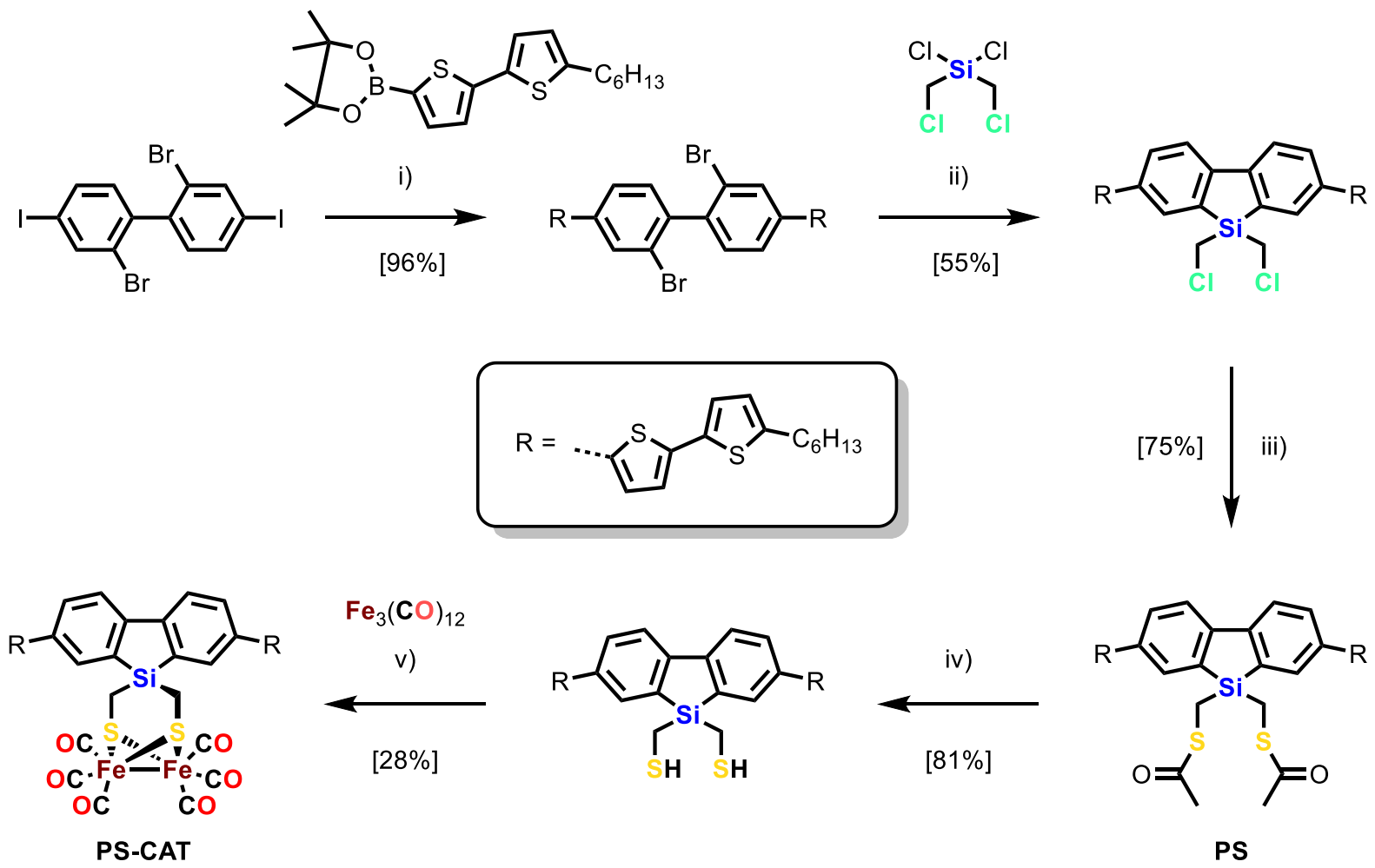

Scheme 1: Synthetic pathway to PS-CAT via PS. i) $\mathrm{BnNEt}_{3} \mathrm{Cl},\left[\mathrm{Pd}\left(\mathrm{PPh}_{3}\right)_{4}\right], \mathrm{K}_{2} \mathrm{CO}_{3}$, toluene; $60{ }^{\circ} \mathrm{C}, 12 \mathrm{~h}$ ii) $n$-BuLi, THF; $-78^{\circ} \mathrm{C}$ to r. t., 12 h iii) $\mathrm{KSCOCH}_{3}$, THF; r. t., 20 h iv) $\mathrm{LiAlH}_{4}, \mathrm{Et}_{2} \mathrm{O}$; $0{ }^{\circ} \mathrm{C}$ to r. t., $\left.12 \mathrm{~h} \mathrm{v}\right) \mathrm{Fe}_{3}(\mathrm{CO})_{12}$, toluene/NMP (20:1); r. t., $12 \mathrm{~h}$.

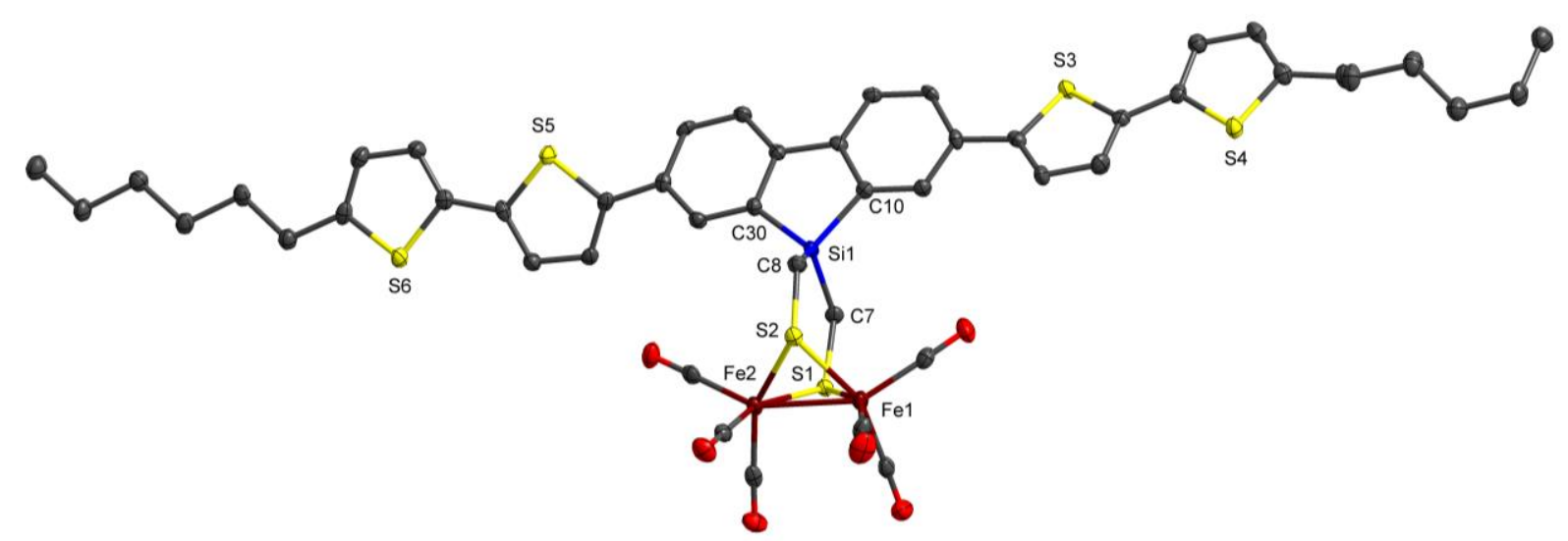

Figure 1: Molecular structure and atom labeling scheme of PS-CAT. The ellipsoids represent a probability of $30 \%$. Hydrogen atoms are omitted for clarity. For selected bond lengths and angles see Table S2.

The photocatalytic proton reduction behaviour of PS-CAT was studied under irradiation with visible light in a 5:1 mixture of $\mathrm{CH}_{3} \mathrm{CN}$ and N-Methyl-2-pyrrolidone (NMP) and with the two electron, one proton sacrificial donor 1,3-dimethyl-2-phenylbenzimidazoline $(\mathrm{BIH})^{[10]}$ (1000 equivalents). The solvent mixture ensures an overall high dielectric constant for good solubility supporting the catalytic performance. Moreover, the weak base NMP can potentially react with the oxidized donor $\left(\mathrm{BIH}^{++}\right)$to capture the proton, thus inhibiting potential back 
electron transfer processes. ${ }^{[11]}$ The photocatalytic reactions were performed in a custom-made, modular 3D printed photoreactor setup (Figure S1). Figure 2 shows the hydrogen evolution time profile. Within the initial 23 hours the catalytic turn-over increases slightly sigmoidally, before the hydrogen evolution reaches a plateau at TON $\approx 210\left(T O F \approx 6.4 \mathrm{~h}^{-1}\right)$. This visiblelight activity is the highest reported for molecular dyads employing [ $\mathrm{FeFe}]$ hydrogenase mimics. ${ }^{[1 d, 4,12]}$ No hydrogen was detected in corresponding experiments without PS-CAT, BIH or both or in the dark. Further photocatalytic results are given in Table S3.

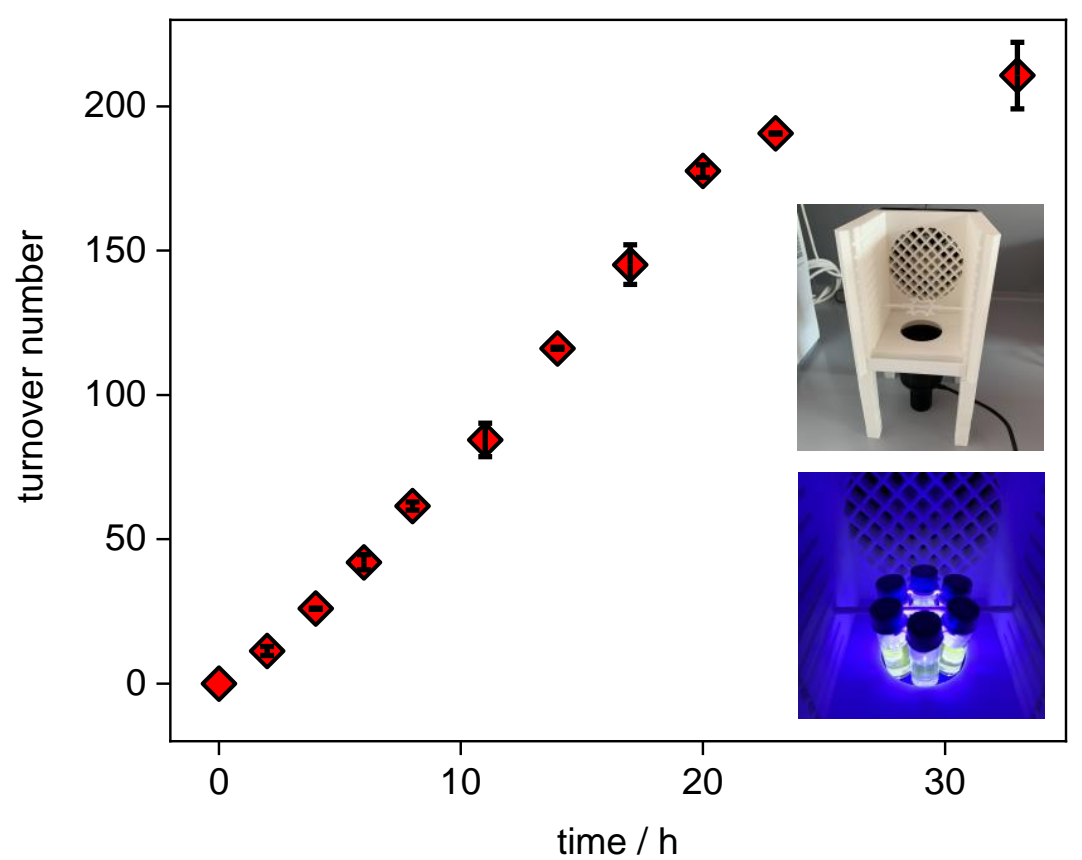

Figure 2: Photocatalytic hydrogen production by PS-CAT $(10 \mu \mathrm{M})$ in $\mathrm{CH}_{3} \mathrm{CN} / \mathrm{NMP}(5: 1)$ with BIH (1000 equivalents) upon irradiation at $455 \mathrm{~nm}$ in a 3D printed photoreactor platform (see inset). Hydrogen was quantified by GC-TCD with samples drawn from the head space. Each point was determined in duplicate and the error bars give the range of variation.

Figure 3a depicts the UV-vis spectra of PS and PS-CAT, which show a strong absorption in the visible spectral region $\left(\lambda_{\max }=409 \mathrm{~nm}, \varepsilon \approx 8.1 \cdot 10^{4} \mathrm{~L} \mathrm{~mol}^{-1} \mathrm{~cm}^{-1}\right.$ for PS and $\lambda_{\max }=410 \mathrm{~nm}$, $\varepsilon \approx 6.1 \cdot 10^{4} \mathrm{~L} \mathrm{~mol}^{-1} \mathrm{~cm}^{-1}$ for PS-CAT) resulting from a $\pi \pi^{*}$ transition localized within the oligothiophene moiety (see $S_{1}$ in Figure $3 d$ ). For PS-CAT TDDFT reveals a dipole allowed charge-transfer transition in the visible region (see $S_{2}$ in Figure $3 d$ ) shifting electron density from a photosensitizer-localized $\pi$ orbital to the [FeFe] hydrogenase mimic unit, i.e. into the $\sigma^{*}$ orbital of the Fe-Fe bond of PS-CAT, contributing to the absorption in the visible range (Figure S12). 
Emission spectra ( $\lambda_{\mathrm{exc}}=410 \mathrm{~nm}$ ) of both PS and PS-CAT display three nearly identical vibronic peaks at 455, 484 and $515 \mathrm{~nm}$ (shoulder) spaced by $1250 \mathrm{~cm}^{-1}$, corresponding to a ground state vibrational mode coupled to the electronic transition (Table S6). The emission lifetimes were determined to $1 \mathrm{~ns}$ and the quantum yields are $\Phi_{\mathrm{F}}=0.54$ in PS and $\Phi_{\mathrm{F}}=0.28$ in PSCAT. The reduced emission quantum yield in PS-CAT are associated with the population of a charge-separated state $\left(S_{2}\right)$ by quantum chemical simulations. $S_{2}$, which is not available in PS, is in close energetic proximity to the $\pi \pi^{*} S_{1}$ state.
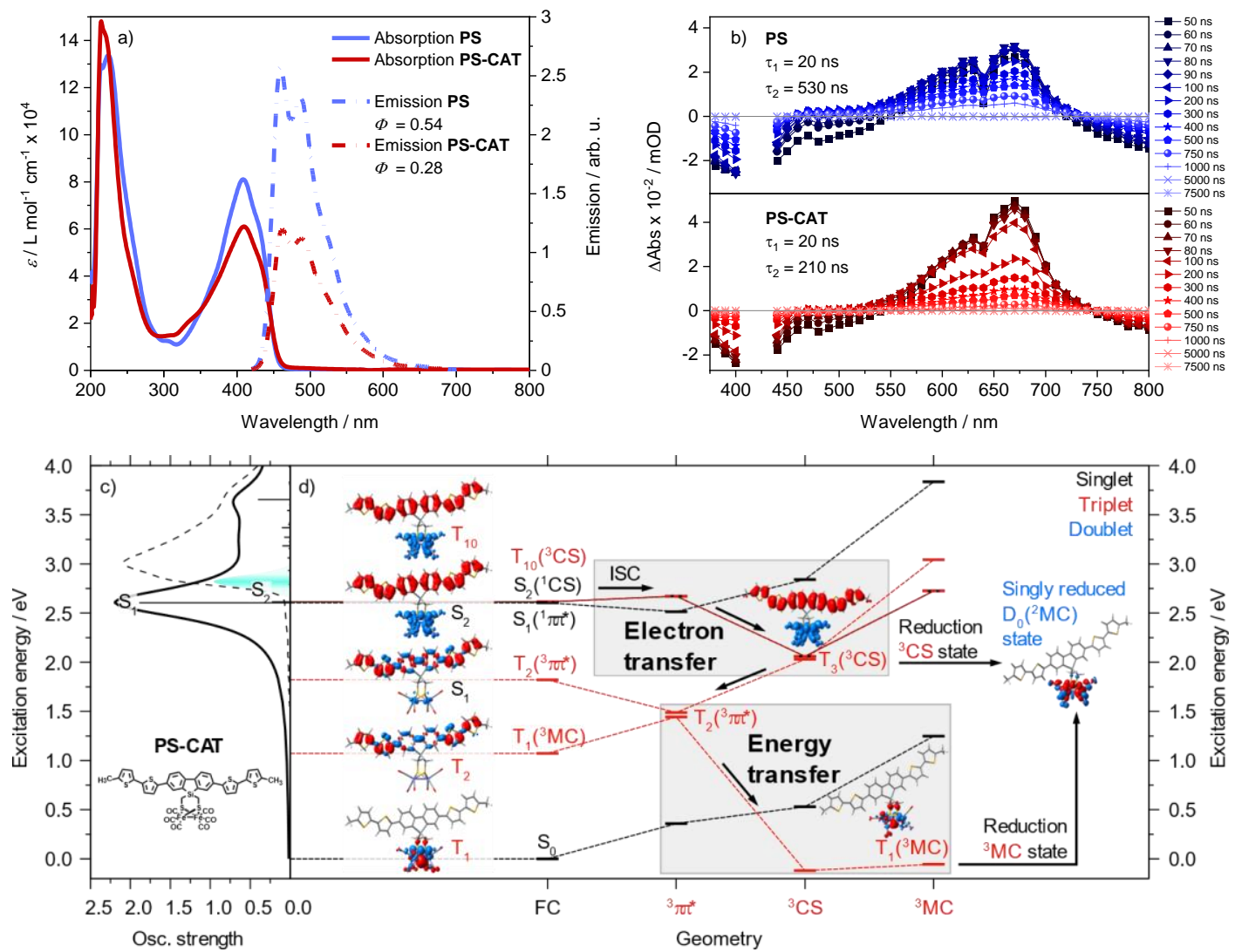

Figure 3: a) Steady-state UV-vis absorption (solid lines) of PS (red) and PS-CAT (blue) and emission (dashed lines) in deaerated THF excited at $410 \mathrm{~nm}$. b) Transient absorption spectra at indicated delay times of PS (top, blue) and PS-CAT (bottom, red) upon excitation at $420 \mathrm{~nm}$ in deaerated THF. c) Experimental (black, dashed) and simulated UV-vis absorption spectrum of PS-CAT in THF; electronic transitions of interest are labelled. d) Potential excited state relaxation cascades associated to electron and energy transfer competing with radiative recombination; relative energies of involved singlet (black) and triplet states (red) are given within their respective equilibrium structures. Electron transfer and energy transfer pathways both leading to singly reduced PS-CAT $\left(\mathrm{D}_{0},{ }^{2} \mathrm{MC}\right.$, blue) upon $\mathrm{BIH}$ reduction are highlighted. Electronic characters are indicated by charge-density differences (CDDs, electronic transitions) and spin densities (opened-shell ground state); charge transfer takes place from red to blue (Table S7). 
Nanosecond-transient absorption spectroscopy was performed to detect the impact of the [FeFe] hydrogenase mimic on the relaxation of the triplet state populated via intersystem crossing (ISC). The data (Figure 3b, Figure S6) for PS and PS-CAT exhibit similar spectral features. Both PS and PS-CAT show a negative signal between 350 and $550 \mathrm{~nm}$, which stems ground-state bleach and emission contributions between 460 and $550 \mathrm{~nm} \cdot{ }^{[13]}$ Excited-state absorption (ESA) is detected at wavelengths longer than $550 \mathrm{~nm}$ for both PS and PS-CAT which is characteristic for the ${ }^{3} \pi \pi^{*}$ state of the sensitizer unit. ${ }^{[14]}$ TDDFT simulations on the dipole-allowed triplet-triplet excitations of PS-CAT, within the equilibrium structure of the ${ }^{3} \pi \pi^{*}$ ground state, see $T_{2}\left({ }^{3} \pi \pi^{\star}\right)$ in Figure $3 \mathrm{~d}$, associate these ESA features to ${ }^{3} \pi \pi^{*}$ transitions of the excited oligothiophene at 600 and $529 \mathrm{~nm}$, respectively (see $T_{11}$ and $T_{16}$ in Figure S14) confirming this assignment. ${ }^{[15]}$ At wavelengths above $\approx 750 \mathrm{~nm}$, a second negative signal can be detected which can be assigned to the triplet phosphorescence of the thiophenes. ${ }^{[15]}$ Both transient signals of PS and PS-CAT decay biexponentially with lifetimes of $\tau_{1}=20 \mathrm{~ns}$ (reflecting the time-resolution of the experimental setup) and $\tau_{2}=530 \mathrm{~ns}$ for PS and $\tau_{1}=20 \mathrm{~ns}$ and $\tau_{2}=210 \mathrm{~ns}$ for PS-CAT. The fast component indicates the decay of the residual emission. The long-lived component is associated with the decay of the ${ }^{3} \pi \pi^{*}$ state of the sensitizer unit. The shortening in $\tau_{2}$ reflects changes in the ${ }^{3} \pi \pi^{*}$ state lifetime of the excited sensitizer in PSCAT indicating the presence of additional relaxation channels occurring from the ${ }^{3} \pi \pi^{*}$ state with an estimated time constant of $348 \mathrm{~ns}$. Nevertheless, besides the changes in lifetime of the triplet state we do not observe any absorption features at 400,580 and $700 \mathrm{~nm}$, which could be ascribed to the reduced [FeFe] hydrogenase according to literature and UV-vis SEC data (Figure S10/S11). Hence, no direct indication for a charge-separated state ( ${ }^{3} \mathrm{CS}$, Figure $\left.3 \mathrm{~d}\right)$ is observed. ${ }^{[13 a, 16]}$ The absence of these features indicates either that no charge transfer takes place to reduce the Fe-Fe unit or that the CS state is too short-lived, recombines quickly and escapes experimental detection in the absence of an electron donor.

Quantum chemical simulations indicate two relaxation pathways for PS-CAT: i) electron transfer causing charge separation and which is accessible from the initially excited singlet states. ii) Energy transfer deactivating the ${ }^{3} \pi \pi^{*}$ state (Figure 3d). The electron transfer pathway leads from the initially excited ${ }^{1} \pi \pi^{*}$ state via ISC to the adjacent charge-separated $\mathrm{T}_{10 / 3}\left(\mathrm{FC} /{ }^{3} \mathrm{CS}\right.$ equilibria) state (Figure 3d), resulting in a pronounced elongation of the Fe-Fe bond (Table S4) of the formally [ $\left.\mathrm{Fe}^{\mathrm{F}} \mathrm{Fe}^{0}\right]$ active site. Upon population of the ${ }^{3} \pi \pi^{*}$ state, either via ${ }^{1} \pi \pi^{*} \rightarrow{ }^{3} \pi \pi^{*}$ ISC or via relaxation from the ${ }^{3} \mathrm{CS}$ state, an energy transfer pathway is available. In this case, hole transfer from the photooxidized photosensitizer to the photoreduced iron cluster yields an excited metal centered $\left({ }^{3} \mathrm{MC}\right)$ state. During this process, the ESA decays as the initial configuration of the oligothiophene-based chromophore is reformed (Figure S14) in agreement with the experimental observations. In this ${ }^{3} \mathrm{MC}$ state, cleavage of the Fe-Fe bond (Table S4) 
occures as both the $\sigma_{\mathrm{Fe}-\mathrm{Fe}}$ and the $\sigma_{\mathrm{Fe}-\mathrm{Fe}}{ }^{*}$ orbitals are singly populated (bond order: 0 ). The population of the ${ }^{3} \mathrm{MC}$ state can be an explanation for the missing signatures of a CS state in the transient absorption experiment. These findings indicate that the presence of sacrificial agent under real catalytic conditions is crucial for the photoinduced reduction of the [FeFe] unit, a key step of the light-induced catalytic process. In this case, according to TDDFT simulations, both relaxation channels, i.e. electron transfer and energy transfer, yield the same singly reduced doublet species of $\left[\mathrm{Fe}^{\prime} \mathrm{Fe}^{0}\right]$ character (see $\mathrm{D}_{0}$ spin density in Figure $3 \mathrm{~d}$ ), blue) - either upon reduction by $\mathrm{BIH}$ of the charge-separated species $\left({ }^{3} \mathrm{CS}\right)$ or upon reduction of the ${ }^{3} \mathrm{MC}$ state. Alternatively, the sacrificial agent could also interfere with the described relaxation processes already in an initial stage of the relaxation cascade reducing the sensitizer directly after excitation. The quenching behaviour of the fluorescence of PS by BIH gives the first indication for such a fast process (Figure S4).

The role of the sacrificial agent is elucidated by operando UV-vis spectroscopy in the presence of sacrificial donor BIH (1000 equivalents) upon irradiation at $455 \mathrm{~nm}$. After 30 minutes of irradiation, the absorption band of PS-CAT at $403 \mathrm{~nm}$ in $\mathrm{CH}_{3} \mathrm{CN} / \mathrm{NMP}$ (5:1) disappeared in favour of a characteristic band at $399 \mathrm{~nm}$, which continues to build up within 17 hours (Figure S3a). Upon irradiation in the absence of the donor the absorption at $403 \mathrm{~nm}$ decays only slowly, indicating the reaction of PS-CAT with BIH (Figure S3c). The newly formed species decomposed slowly in the dark and quite rapidly in the presence of oxygen (Figure S3b). It is tentatively attributed to a sensitive follow-up product of the catalytic species, not to the catalytic species itself.

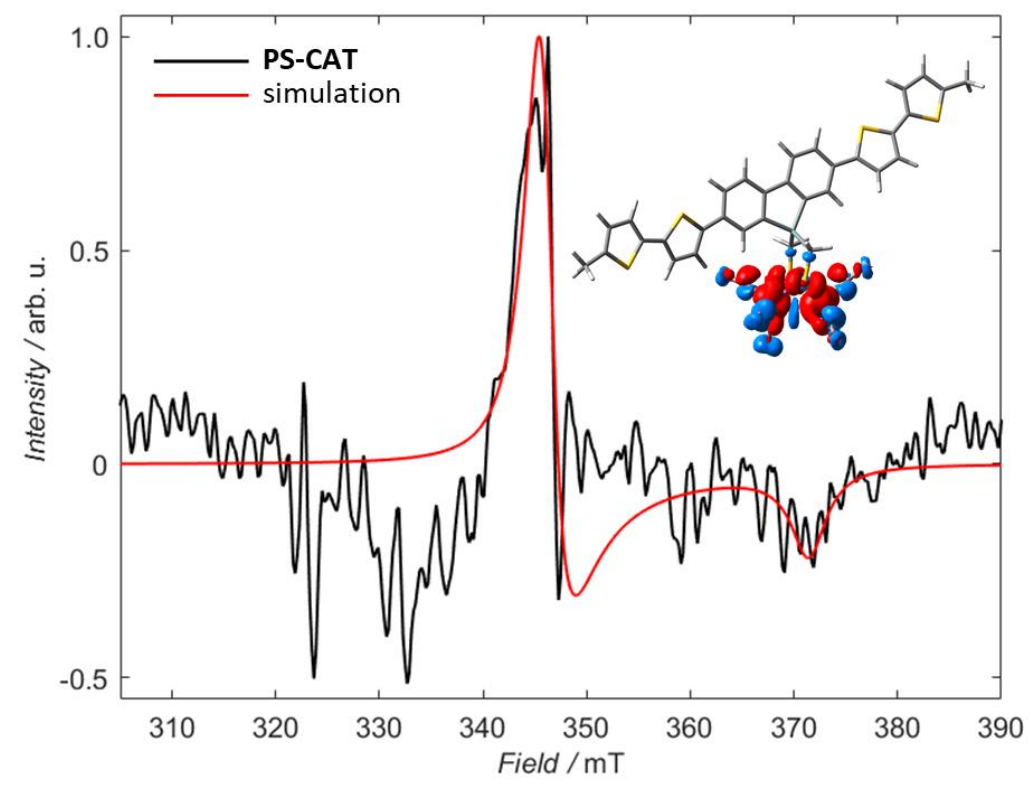


Figure 4: EPR spectrum of PS-CAT after four hours of illumination under catalytic conditions (30 $\mu \mathrm{M}$ PS-CAT). Illumination was carried out in a MD5 resonator and the EPR measurement was done at $4 \mathrm{~K}$. The negative signal at $330 \mathrm{mT}$ stems from resonator background. Inset: Spin density localized at the [FeFe] moiety of the singly reduced PS-CAT (doublet).

Operando electron paramagnetic resonance (EPR) spectroscopy reveals the formation of a reduced [FeFe] moiety under photocatalytic conditions. The catalytic mixture (30 $\mu \mathrm{M}$ PS-CAT, 1000 equivalents $\mathrm{BIH}$ ) was illuminated in the resonator at $270 \mathrm{~K}$ and measured at $4 \mathrm{~K}$. The data show the formation of a paramagnetic [ $\left.\mathrm{Fe}^{\prime} \mathrm{Fe}^{0}\right]$ intermediate (Figure 4), which has been postulated as key species in the light-induced HER mechanism. ${ }^{[6 c]}$ No signal was detected in the dark or at room temperature (Figure S7a). The $g$ values $\left(g_{1}=2.003, g_{2}=1.99557\right.$, $\left.g_{3}=1.86197\right)$ derived from the EPR data are similar to those reported on $\left[\mathrm{Fe}^{\prime} \mathrm{Fe}^{0}\right]$ species with the third $g$ tensor being shifted further low field. ${ }^{[17]}$ This shift could be due to a low concentration of the $\left[\mathrm{Fe}^{\prime} \mathrm{Fe}^{0}\right]$ intermediate in photochemical reduction, consequently leading to a lower EPR spectral resolution compared to that resulting from (electro)chemical reduction processes. ${ }^{[17-}$ 18]

In summary, we synthesized a precious metal-free photocatalyst dyad PS-CAT for photocatalytic hydrogen generation under visible light. The dyad as prototype comprising $\pi$ conjugated oligothiophenes as light absorbers shows a remarkable, long-term photocatalytic activity, the best reported for comparable complexes in the visible spectral range. In accordance with theory, operando EPR spectroscopy using BIH as sacrificial donor reveals the generation of an active $\left[\mathrm{Fe}^{\prime} \mathrm{Fe}^{0}\right]$ species, which presumably drives the light-induced hydrogen generation. 


\section{Acknowledgements}

M.K.F. thanks the "Katholischer Akademischer Ausländer-Dienst" (KAAD) for the scholarship. C.K. is grateful to the "Deutscher Akademischer Austauschdienst" (DAAD) for an internship (ERASMUS+). The authors thank Professor C. Robl for helpful discussions and S. Benndorf for recording IR SEC spectra. Professor C. Kay is thanked for valuable discussion on the EPR measurements. We are grateful to $\mathrm{H}$. Elbeheiry for the preparation of the Table of Contents. Financial support by the German Science Foundation via the TRR234 CataLight is gratefully acknowledged (project number 364549901, projects A2, C5 and Z2). All calculations were performed at the Universitätsrechenzentrum of the Friedrich Schiller University Jena.

Keywords: Photocatalytic hydrogen evolution - Quantum chemistry • [FeFe] hydrogenase • Spectroelectrochemistry • Electron paramagnetic resonance (EPR) spectroscopy • Conjugated oligothiophenes • Photosensitizer-catalyst dyad • Time-resolved spectroscopy 


\section{References}

[1] a) A. J. Esswein, D. G. Nocera, Chem. Rev. 2007, 107, 4022-4047; b) L.-Z. Wu, B. Chen, Z.-J. Li, C.-H. Tung, Acc. Chem. Res. 2014, 47, 2177-2185; c) Y.-J. Yuan, Z.-T. Yu, D.-Q. Chen, Z.-G. Zou, Chem. Soc. Rev. 2017, 46, 603-631; d) W. T. Eckenhoff, Coord. Chem. Rev. 2018, 373, 295-316.

[2] W. Lubitz, H. Ogata, O. Ruediger, E. Reijerse, Chem. Rev. 2014, 114, 4081-4148.

[3] a) C. Tard, C. J. Pickett, Chem. Rev. 2009, 109, 2245-2274; b) T. R. Simmons, G. Berggren, M. Bacchi, M. Fontecave, V. Artero, Coord. Chem. Rev. 2014, 270-271, 127150.

[4] a) K. E. Dalle, J. Warnan, J. J. Leung, B. Reuillard, I. S. Karmel, E. Reisner, Chem. Rev. 2019, 119, 2752-2875; b) J. Amaro-Gahete, M. V. Pavliuk, H. Tian, D. Esquivel, F. J. Romero-Salguero, S. Ott, Coord. Chem. Rev. 2021, 448, 214172.

[5] K. Hola, M. V. Pavliuk, B. Nemeth, P. Huang, L. Zdrazil, H. Land, G. Berggren, H. Tian, ACS Catal. 2020, 10, 9943-9952.

[6] a) R. Goy, U.-P. Apfel, C. Elleouet, D. Escudero, M. Elstner, H. Görls, J. Talarmin, P. Schollhammer, L. Gonzalez, W. Weigand, Eur. J. Inorg. Chem. 2013, 2013, 4466-4472; b) R. Goy, L. Bertini, H. Görls, L. De Gioia, J. Talarmin, G. Zampella, P. Schollhammer, W. Weigand, Chem. - Eur. J. 2015, 21, 5061-5073; c) R. Goy, L. Bertini, T. Rudolph, S. Lin, M. Schulz, G. Zampella, B. Dietzek, F. H. Schacher, L. De Gioia, K. Sakai, W. Weigand, Chem. - Eur. J. 2017, 23, 334-345.

[7] a) A. Mishra, P. Bäuerle, Angew. Chem. Int. Ed. 2012, 51, 2020-2067; b)A. Mishra, P. Bäuerle, Angew. Chem. 2012, 124, 2060-2109.

[8] A. Mishra, M. K. R. Fischer, P. Bäuerle, Angew. Chem. Int. Ed. 2009, 48, 2474-2499.

[9] M. G. Gatty, S. Pullen, E. Sheibani, H. Tian, S. Ott, L. Hammarström, Chem. Sci. 2018, 9, 4983-4991.

[10] X.-Q. Zhu, M.-T. Zhang, A. Yu, C.-H. Wang, J.-P. Cheng, J. Am. Chem. Soc. 2008, 130, 2501-2516.

[11] Y. Tamaki, K. Koike, T. Morimoto, O. Ishitani, J. Catal. 2013, 304, $22-28$.

[12] a) H.-H. Cui, M.-Q. Hu, H.-M. Wen, G.-L. Chai, C.-B. Ma, H. Chen, C.-N. Chen, Dalton Trans. 2012, 41, 13899-13907; b) A. Mazzeo, S. Santalla, C. Gaviglio, F. Doctorovich, J. Pellegrino, Inorg. Chim. Acta 2021, 517, 119950.

[13] a) Y. Na, J. Pan, M. Wang, L. Sun, Inorg. Chem. 2007, 46, 3813-3815; b) Y. Na, M. Wang, J. Pan, P. Zhang, B. Akermark, L. Sun, Inorg. Chem. 2008, 47, 2805-2810.

[14] a) Z. Xu, T. Jin, Y. Huang, K. Mulla, F. A. Evangelista, E. Egap, T. Lian, Chem. Sci. 2019, 10, 6120-6124; b) S. Rentsch, J. P. Yang, W. Paa, E. Birckner, J. Schiedt, R. Weinkauf, Phys. Chem. Chem. Phys. 1999, 1, 1707-1714; c) J. P. Yang, W. Paa, S. Rentsch, Synth. Met. 1999, 101, 624-625.

[15] D. Wasserberg, P. Marsal, S. C. J. Meskers, R. A. J. Janssen, D. Beljonne, J. Phys. Chem. B 2005, 109, 4410-4415.

[16] S. J. Borg, T. Behrsing, S. P. Best, M. Razavet, X. Liu, C. J. Pickett, J. Am. Chem. Soc. 2004, 126, 16988-16999.

[17] P. S. Singh, H. C. Rudbeck, P. Huang, S. Ezzaher, L. Eriksson, M. Stein, S. Ott, R. Lomoth, Inorg. Chem. 2009, 48, 10883-10885.

[18] a) M. Razavet, S. J. Borg, S. J. George, S. P. Best, S. A. Fairhurst, C. J. Pickett, Chem. Commun. 2002, 700-701; b) A. Silakov, J. L. Shaw, E. J. Reijerse, W. Lubitz, J. Am. Chem. Soc. 2010, 132, 17578-17587; c) D. Streich, M. Karnahl, Y. Astuti, C. W. Cady, L. Hammarström, R. Lomoth, S. Ott, Eur. J. Inorg. Chem. 2011, 2011, 1106-1111. 